\title{
Unusual CD5+ large B-cell lymphoma: Possible follicular variant of diffuse large $B$-cell lymphoma
}

\author{
Sainan Wei • Brian Olsen
}

Received: 24 November 2011 / Accepted: 9 May 2012 /Published online: 29 May 2012

(C) Springer-Verlag 2012

\begin{abstract}
We report the unusual case of a CD5+ large B-cell lymphoma involving lymph node, peripheral blood and the bone marrow of a 63-year-old female. Lymph node findings included an exclusive population of large neoplastic cells against a background of well-formed follicular dendritic cell meshworks. Immunophenotypic findings were reminiscent of those seen in small lymphocytic lymphoma/ chronic lymphocytic leukemia (SLL/CLL), including CD5 and CD23 expression. There were no immunophenotypic or genotypic findings to suggest mantle cell lymphoma or conventional follicular lymphoma. There was a remote history of a CD5+ "diffuse large B-cell lymphoma" from biopsy of an abdominal mass, with an involved bone marrow, monotypic for the same light chain as the more recent specimen. This lesion displayed a virtually identical histology, and was also found to be CD23 positive. Concordant bone marrow involvement was documented in both 1993 and 2004. A relationship to SLL/CLL was never documented at any point in the patients' clinical course, but is difficult to exclude. Alternatively, findings may be more consistent with a follicular variant of diffuse large B-cell lymphoma, CD5+.
\end{abstract}

Keywords CD5+ large B-cell lymphoma · Lymph node · Chronic lymphocytic leukemia/small lymphocytic lymphoma $\cdot$ Follicular variant

\footnotetext{
S. Wei East Lansing, MI, USA

B. Olsen $(\bowtie)$

Ingham Regional Medical Center,

Lansing, MI 48910, USA

e-mail: brian.olsen@irmc.org
}

Division of Human Genetics, Michigan State University,

\section{Introduction}

Follicular lymphomas are associated with characteristic pathologic findings [1]. These include varying proportions of small and large lymphocytes, expressing pan B-cell markers, CD10 and BCL6, with aberrant expression of BCL2. Most have a predominantly follicular growth pattern, including a background of follicular dendritic cell meshworks, the latter definable by expression of markers such as CD21 and CD23. However, diffuse areas may also be present, in varying proportion, including rare cases having an entirely diffuse architecture. Grade is assigned based on the proportion of large cells, or centroblasts present, with lesions further characterized by a lack of admixed small cells, or centrocytes, designated as grade $3 b$. Importantly, the presence of diffuse areas including a predominance of large cells merits the separate, or alternative, diagnosis of diffuse large B-cell lymphoma (DLBCL). The majority of cases harbor the $t(14 ; 18)$ resulting in juxtaposition of the BCL2 and IgH genes, and BCL2 overexpression.

Occasional cases deviate from the expected immunophenotype or genotype. Such alterations include lack of CD10 expression, BCL2 expression or the $t(14 ; 18)$, and these variations are reported more commonly in grade 3 lesions, particularly grade $3 \mathrm{~b}[1]$. CD5 expressing follicular lymphomas have also been reported. Although these may be over represented among the so-called "floral variant" of follicular lymphoma, these cases generally display the expected immunophenotype and genotype including the $t(14 ; 18)$. Furthermore, they have been reported to lack additional features associated with potential mimics, such as chronic lymphocytic leukemia (CLL) and mantle cell lymphoma, including lack of cyclin D1, CD43 or IgD expression $[2,3]$.

Roughly $10 \%$ of diffuse large B-cell lymphomas (DLBCL), not otherwise specified express CD5. The majority 
represents de novo cases, and share additional immunophenotypic and genotypic features with, so-called, activated B-cell-like DLBCL; these also share a relatively poor clinical outcome. These can be distinguished from the blastoid variant of mantle cell lymphoma by an absence of cyclin D1 expression $[4,5]$. A minor proportion of CD5 positive DLBCL arise secondarily, most from preexistent chronic lymphocytic leukemia/small lymphocytic lymphoma (CLL/SLL), but occasionally from other small B-cell lymphomas, including rare reports of origin from $\mathrm{CD} 5$ positive follicular lymphoma [6].

We report an unusual case, that of a CD5+ large B-cell lymphoma presenting in the background of well-defined follicular dendritic meshworks, and briefly discuss the clinical and pathologic findings in the context of known entities, particularly CLL, with special reference to Richter syndrome, and conventional follicular lymphoma.

\section{Clinical history}

A female patient presented to medical attention in 1993, at the age of 63, with abdominal pain. CT scanning revealed marked intra-abdominal lymphadenopathy. An axillary lymph node biopsy was said to yield a diagnosis of DLBCL, CD5 positive and lambda monotypic by flow cytometry. A subsequent bone marrow biopsy was described as positive for involvement by DLBCL, also CD5 positive and lambda monotypic by flow cytometry. Peripheral blood findings were not commented on in the bone marrow biopsy report. She had no B symptoms, and both review of systems and past medical history were otherwise unremarkable. She was treated with eight cycles of CHOP chemotherapy, was said to achieve a complete remission, and was well for roughly 10 years. In March 2004, she presented with left inguinal lymphadenopathy, with small lymph nodes palpable in the left supraclavicular area and left axilla. CT scanning showed retrocrural, retroperitoneal, inguinal, porta hepatis, bilateral cervical and mesenteric root lymphadenopathy, with the largest conglomerate of intra-abdominal lymph nodes measuring $8.9 \mathrm{~cm}$. in greatest dimension. Abnormal circulating cells were apparent, following morphologic review of peripheral blood. A left inguinal lymph node was biopsied and, subsequently, the bone marrow.

\section{Materials and methods}

Lymph node biopsy material from 2004 was fixed in $10 \%$ neutral buffered formalin, routinely processed, cut into $5-\mu \mathrm{m}$ sections, and stained with hematoxylin and eosin (HE). A bone marrow biopsy, also from 2004, was fixed in $10 \%$ neutral buffered formalin, decalcified in Formical-4 (formic acid, formaldehyde, EDTA and deionized water), routinely processed, cut into 5 - $\mu \mathrm{m}$ sections, and HE stained. Bone marrow aspirate smears were prepared manually and stained with May-Grunwald Giemsa. A peripheral blood smear from 2004 was prepared manually and Wright stained. Immunohistochemistry was performed on lymph node and bone marrow biopsy sections, cut at $4 \mu \mathrm{m}$. Studies were performed on an automated Ventana Benchmark XT immunostainer, employing a streptavidin-biotin immunoperoxidase technique. The following antibodies were utilized: BCL2 (Dako; clone 124, undiluted), BCL6 (Dako; clone PG-B6p, diluted 1:10), CD3 (Ventana; clone 2GV6, undiluted), CD5 (Ventana; clone SP19, undiluted), CD10 (Ventana; clone 56 C6, undiluted), CD20 (Dako; clone L26, undiluted), CD21 (Dako; clone 1 F8, diluted 1:25), CD43 (Ventana; clone L60, undiluted), CD79a (Ventana; clone JCB117, undiluted), cyclin D1 (Ventana; clone SP4, undiluted), IgD (Cell Marque; polyclonal, undiluted), Ki-67 (Ventana; clone 30-9, undiluted), MUM1 (Dako; clone MUM1p, diluted 1:50). A reagent combining ethylenediaminetetraacetic acid, boric acid and Tris buffer was used for antigen retrieval for all antibodies (CCI reagent; Ventana), with the exception of $\mathrm{CD} 21$, for which an alternative reagent was used (protease 1 reagent, Ventana). Only CD3 and CD20 were applied to bone marrow biopsy sections. All antibodies listed were applied to lymph node biopsy sections.

Conventional cytogenetic analysis of bone marrow aspirate from 2004 was performed as follows: direct harvest was undertaken the same day the sample was received. Shortterm unstimulated and 12-O-tetradecanoylphorbol-13-acetate (TPA) stimulated cell cultures were harvested 24 and $96 \mathrm{~h}$, respectively, after the cultures were set up. Chromosome analyses were performed on metaphase cells that were Giemsa banded with trypsin using Leischmann stain (GTL banded). Lymph node from 2004 was studied by fluorescence in situ hybridization (FISH), performed on interphase cells with single color probes D12Z3 (12 centromere), D13S319 and LAMP (13q14.3 and D13q34), p53 (17p13.3) and ATM (11q22.3), as well as dual color dual fusion probes CCND1 (11q13) and IgH (14q32), BCL2 (18q21) and IgH (14q32)(Vysis, Inc.). At least 200 interphase cells were scored for each probe.

A sample of peripheral blood from 2004 was sent to an outside laboratory for flow cytometric study following which a report was received. Findings are summarized in the Results section.

Subsequent to the above, pathologic materials from 1993 were requested from the outside institution where these studies had been performed. A representative HE stained section of axillary lymph node was made available, as were unstained sections. The latter were stained for CD20, CD5, $\mathrm{CD} 21$ and CD23, under similar conditions as those described above. Results of a complete blood count performed at the time of bone marrow biopsy were also made available. A bone marrow aspirate smear and HE stained biopsy section were provided. A peripheral blood film was not 
provided, nor were detailed results of flow cytometric immunophenotyping.

\section{Results}

Lymph node, peripheral blood and bone marrow findings from 2004 are discussed. Lymph node architecture was effaced by an atypical lymphoid infiltrate, including a predominance of cells of intermediate to large size (Fig. 1a and b), having irregular nuclear contours, a vesicular chromatin pattern, one or more prominent nucleoli, and moderately abundant, pale eosinophilic cytoplasm. A follicular pattern was suggested by HE stained histologic sections (Fig. 2a). This impression was also supported by the pattern of expression of B-cell associated markers such as CD79a and CD20 (Fig. 2b) by immunohistochemistry. CD21 immunostaining revealed well-defined follicular dendritic cell meshworks, throughout the lesional tissue (Fig. 2c). Large cells were also predominant in interfollicular areas. There was a minor, background population of admixed small lymphocytes, lacking atypia, and consisting predominantly of T-cells (CD3+). By immunohistochemistry, the large cells also expressed BCL2, CD5, CD23, CD43 and MUM-1, included a low proportion of Ki-67 expressing cells, estimated at $5-10 \%$, and lacked expression of cyclin D1. The large cells also failed to express CD10, BCL6 and IgD. There was no pattern of staining with CD10, BCL6 or Ki-67 to suggest persistence of a non-neoplastic follicle center cell population.

Large cells with comparable morphology were also evident in peripheral blood (Fig. 1c). These were found in small numbers. Flow cytometric immunophenotyping revealed a subpopulation of B-cells having light scatter characteristics consistent with large cells, displaying monotypic surface lambda expression, and features similar to those demonstrated by immunohistochemistry (CD19+, CD20+, CD5+, CD23+, CD38+, CD10-, FMC7-). A population of small cells, of similar immunophenotype, was not identified by flow cytometry. The absolute lymphocyte count in peripheral blood was $1.9 \times 10^{9} / 1$ and atypical-appearing small lymphocytes were not identified by morphologic review. Bone marrow biopsy was also performed. Scattered, nodular aggregates of CD20+ large B-cells were identified. Cytogenetic analysis performed on the bone marrow aspirate material revealed a normal female karyotype. Formalin fixed, paraffin embedded lymph node biopsy material was studied by interphase FISH. This study was negative for BCL2/IgH and CCND1/IgH fusion, employing dual fusion probe sets, and negative for $13 \mathrm{q} 14,17 \mathrm{p} 13$ and $11 \mathrm{q} 23$ deletions, as well as trisomy 12 , utilizing locus specific probes.

A representative, HE-stained histologic section of axillary lymph node from 1993 had a similar appearance to that of the 2004 sample, including large cell predominance, and
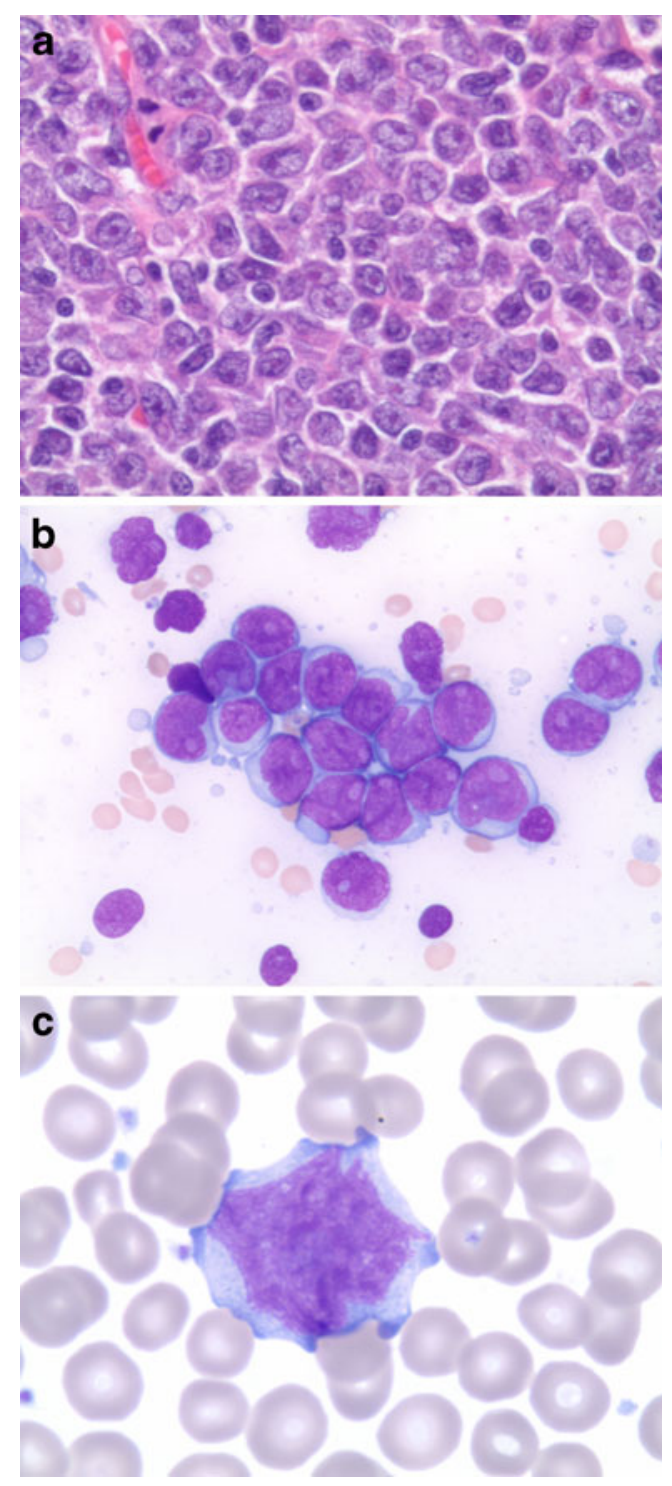

Fig. 1 Large cells were predominant in samples obtained in 2004: a representative image of lymph node (HE stain, $\times 400$ original magnification); b touch imprint of lymph node (May Grunwald Giemsa stain, $\times 400$ original magnification); $\mathbf{c}$ large lymphoma cells found in peripheral blood, comparable to those found in lymph node (Wright stain, $\times 1,000$ original magnification)

the impression of a follicular architecture (Fig. 3a and b). Only a limited amount of tissue remained in the deeper, unstained sections provided, these including only 30-40\% of the tissue surface area present in the original HE stained section. However, CD20, CD5 and CD23 expression by lymphoma cells were clearly evident. CD21 positive follicular dendritic cell meshworks were also evident though, in that portion of the original tissue sample remaining, these appeared attenuated, relative to the 2004 sample.

Histologic sections of bone marrow from 1993 revealed distinct nodules of concordant involvement by lymphoma, with a predominance of large cells, in a pattern very similar to that 

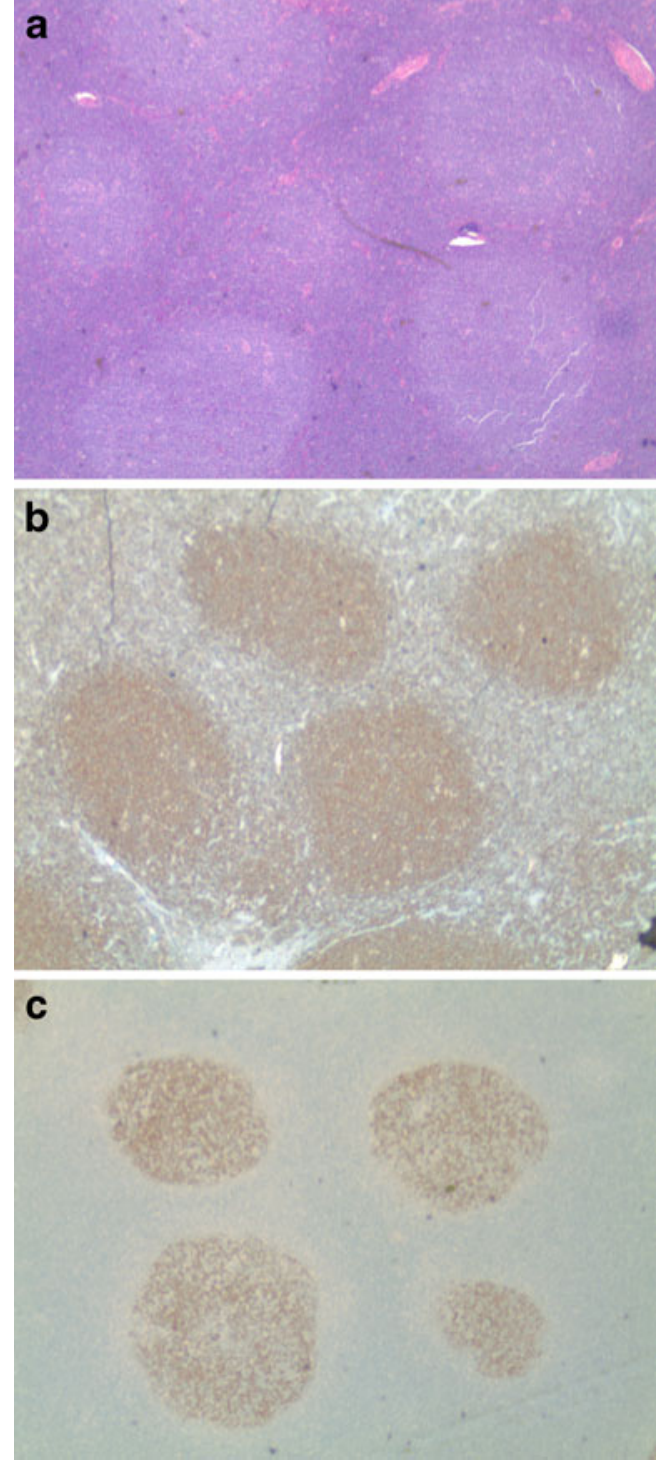

Fig. 2 A follicular architecture was apparent in histologic sections of the 2004 lymph node sample, supported by the following: a HE stain ( $\times 20$ magnification). b CD20 ( $\times 20$ magnification). c CD21 ( $\times 20$ original magnification)

observed in 2004. With some difficulty, atypical large cells were also discernible in the marrow aspirate smears provided. Increased numbers of small lymphocytes were not apparent. Among CBC findings, the WBC count was $6.4 \times 10^{9} / 1$. The automated differential count included lymphocytes of $16 \%$, while the manual differential reported lymphocytes of $22 \%$. There was no report of abnormal-appearing lymphocytes.

\section{Discussion}

In summary, features include those of a CD5+ large B-cell lymphoma, presenting against a background of apparently
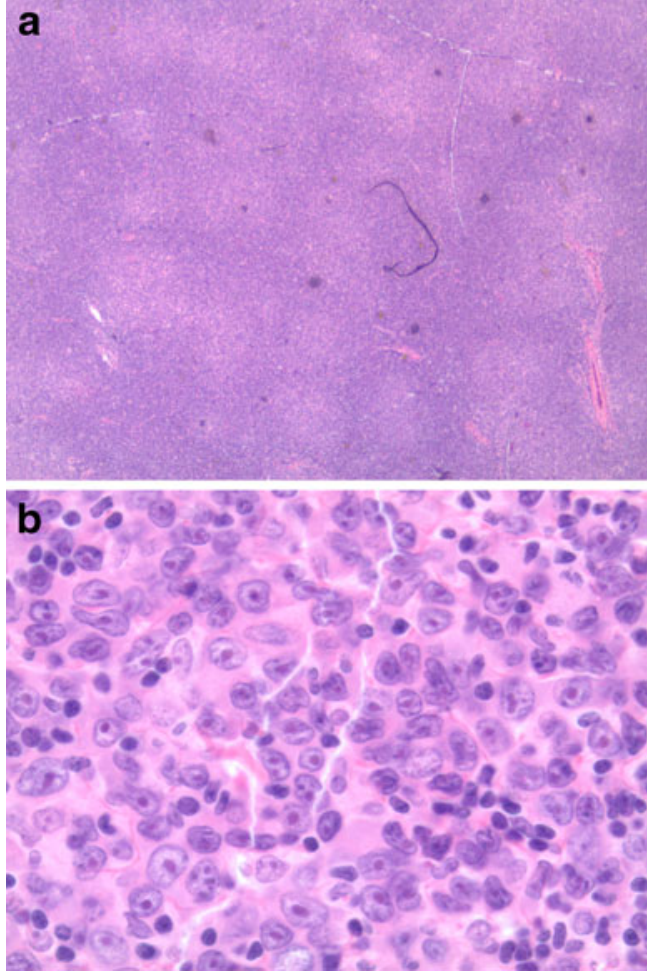

Fig. 3 Representative images from the 1993 lymph node sample, HE stain: a $\times 20$ original magnification. $\mathbf{b} \times 400$ original magnification

undisturbed, well-formed follicular dendritic cell meshworks, diagnosed in 2004. Large lymphoma cells were found in peripheral blood, and there was concordant bone marrow involvement. Though clonal relatedness could not be formally assessed, a lesion of similar histology and immunophenotype had been diagnosed in 1993, presenting with extensive lymphadenopathy, also with concordant bone marrow involvement, and responding favorably to chemotherapy at that time. The immunophenotype in each of the 1993 and 2004 samples shared features reminiscent of CLL, best documented in 2004. In each case, large cells were dominant, with no population of CLL-like small cells suggested by $\mathrm{CBC}$ data, morphologic review of peripheral blood and bone marrow, or flow cytometric immunophenotyping. The patient was also said to achieve a complete remission following a course of chemotherapy in 2004, but has since suffered additional disease relapse, which became refractory to chemotherapy. She eventually succumbed to her disease in June of 2009, roughly 16 years following her initial diagnosis. Immunophenotypic and molecular cytogenetic features to suggest a relationship to either conventional follicular lymphoma, or mantle cell lymphoma are lacking.

The possibility of a Richter type transformation of CLL was suggested at the time of initial evaluation in 1993. However, preexistent or coexistent CLL, essentially a requirement for diagnosis of Richter syndrome as currently 
defined [7], was not documented at that time, or evident in pathologic material at disease relapse. Furthermore, a distinction has been made between clonally related Richter syndrome - representing the majority of cases, these associated with genetic complexity, including TP53 disruptions, and poor prognosis - and clonally unrelated cases - the minority, these lacking genetic complexity, less likely to carry TP53 disruptions, and having a more favorable prognosis, analogous to de novo DLBCL [8]. Though the case under discussion bears some resemblance to a clonally related Richter syndrome, given the CLL-like immunophenotype of lymphoma cells, the prolonged survival is not a typical feature. In addition, TP53 deletion was not apparent, at the level of resolution of interphase FISH. A paraimmunoblastic variant of CLL/SLL would also enter into the differential diagnosis. However, the presence of cells reminiscent of either paraimmunoblasts or prolymphocytes was not a feature of specimens reviewed from either 1993 or 2004 , and the reported behavior of this rare variant is also relatively aggressive [9].

Moreover, well-defined follicular dendritic cell meshworks are not a feature of lymph nodes involved by CLL, and would not be expected as a feature of CLL $[10,11]$ or other low-grade B-cell non-Hodgkin's lymphoma (NHL) in transformation, with the possible exception of follicular lymphoma. Also noteworthy among low grade B-cell NHL in this regard is nodal marginal zone lymphoma, in which follicular colonization by lymphoma cells can be a prominent feature [12]. However, though described, CD5 expression is unusual [13], and transformation generally implies sheeting out of large cells, with loss of a follicular architecture [14]. Nevertheless, a possible relationship to CLL is intriguing, and cannot be entirely excluded, particularly given the immunophenotype of lymphoma cells in this case. Such a relationship may be possible to establish, but would likely require more sophisticated tools than those employed in routine diagnostics, for example, gene expression profiling. Recently, it has been suggested that de novo CD5+ DLBCL may arise from the same progenitor cell as the mutated variant of CLL, with de novo CD5+ DLBCL the result of a pattern of genetic alterations distinct from those typically resulting in CLL, occurring prior to clonal expansion [15].

Large cells were predominant within follicular structures and in interfollicular areas in the 2004 lymph node, and a similar large cell predominance was evident within and outside of putative follicular structures in the 1993 lymph node specimen. This may represent a spillover phenomenon, as is often seen in conventional follicular lymphoma. Alternatively, this finding could be interpreted as indicative of follicular colonization. However, the latter possibility is difficult to rationalize with the observation that follicular dendritic cell meshworks appear undisturbed, as best depicted in the 2004 specimen, of questionable likelihood following invasion by large lymphoma cells.

There has been recent emphasis on the role of the microenvironment in lymphomagenesis. Analogous to conventional follicular lymphoma, it may be that a unique microenvirnonment, as evidenced in part by follicular dendritic cell meshworks, may have played a significant role in sustaining tumor growth in this case, despite a low proliferative index. This contrasts with the usual large B-cell lymphoma, in which proliferative capacity is increased, such that a comparable dependence on the microenvironment is not apparent [16]. A further consequence of this distinction may have been a relatively prolonged survival despite other pathologic indicators of potential adverse prognosis, such as large cell morphology, concordant bone marrow involvement and peripheralized disease.

The unique features of follicular lymphoma, grade 3B, as compared to follicular lymphoma of low histologic grade, and follicular lymphoma, grade $3 \mathrm{~A}$ have also been recently highlighted. These include a lack of CD10 expression and the $\mathrm{t}(14 ; 18)$, with MUM1 expression and BCL6 translocations more commonly observed, among other features more akin to DLBCL. There has been speculation that such cases may in fact represent a "follicular variant" of DLBCL [17]. This apparent contradiction in terms is not without precedent in hematopathology, a noteworthy example being the aforementioned diffuse variant of follicular lymphoma, formally acknowledged in the most recent World Health Organization (WHO) classification scheme. It is conceivable that the current case may also represent a type of follicular variant of DLBCL, albeit with a clinical course more in keeping with a B lymphoid neoplasm of low histologic grade.

Acknowledgements The authors wishes to acknowledge Drs. Marilyn Viera, Elizabeth Layhe, and Martin Bury for providing clinical information pertinent to this case. We also wish to recognize CAP Lab, Lansing, Michigan, for performance of flow cytometric studies (2004), Sparrow Hospital Department of Pathology, for performance of some of the immunohistochemical stains performed (lymph node from 1993), and the Mayo Clinic, Rochester, MN, for sharing pathologic materials and reports from 1993.

Conflict of interest The authors declare that they have no conflict of interest.

\section{References}

1. Swerdlow SH, Campo E et al (2008) WHO classification of tumours of the haematopoietic and lymphoid tissues. IARC, Lyon, pp 220-226 
2. Barry TS, Jaffe ES et al (2002) CD5+ follicular lymphoma - a clinicopathologic study of three cases. Am J Clin Pathol 118:589598

3. Tiesinga JJ, Wu D et al (2000) CD5+ Follicular lymphoma immunophenotyping detects a unique subset of "floral" follicular lymphoma. Am J Clin Pathol 114:912-921

4. Yamaguchi M, Nakamura N et al (2008) De novo diffuse large Bcell lymphoma: results of a detailed clinicopathological review in 120 patients. Haematologica 93:1195-1202

5. Tagawa H, Suguru M et al (2005) Comparison of genome profiles for identification of distinct subgroups of diffuse large B-cell lymphoma. Blood 106:1770-1777

6. Vassallo J, Bousquet M et al (2007) CD5-positive diffuse large Bcell lymphoma arising from a CD5-positive follicular lymphoma. J Clin Pathol 60:573-575

7. Rossi D, Gaidano G (2009) Richter syndrome: molecular insights and clinical perspectives. Hematol Oncol 27:1-10

8. Rossi D, Spina V et al (2011) The genetics of Richter syndrome reveals disease heterogeneity and predicts survival after transformation. Blood 117:3391-3401

9. Pugh W, Manning J et al (1988) Paraimmunoblastic variant of small lymphocytic lymphoma/leukemia. Am J Surg Pathol 12:907-917
10. Schmid C, Isaacson PG (1994) Proliferation Centres in B-cell malignant lymphoma, lymphocytic (B-CLL): an immunophenotypic study. Histopathology 24:445-451

11. Feller AC, Diebold J (2004) Histopathology of nodal and extranodal non-Hodgkin's lymphomas (based on the WHO Classification), 3rd edn. Springer-Verlag, Berlin, pp 23-29

12. Naresh KN (2008) Nodal marginal zone B-cell with prominent follicular colonization - difficulties in diagnosis: a report of 15 cases. Histopathology 52:331-339

13. Jevremovic D, Dronca RS et al (2010) CD5+ B-cell lymphoproliferative disorders: beyond chronic lymphocytic leukemia and mantle cell lymphoma. Leuk Res 34:1235-1238

14. Arcaini L, Lucioni M et al (2009) Nodal marginal zone lymphoma: current knowledge and future directions of a heterogeneous disease. Eur J Haematol 83:165-174

15. Katzenberger T, Lohr A et al (2003) Genetic analysis of de novo CD5+ diffuse large B-cell lymphoma suggests an origin from a somatically mutated CD5+ progenitor B-cell. Blood 101:699-702

16. de Jong D (2005) Molecular pathogenesis of follicular lymphoma: a cross talk of genetic and immunologic factors. J Clin Oncol 23:6358-6363

17. Salaverria I, Siebert R (2011) Follicular lymphoma grade 3B. Best Pract Res Clin Haematol 24:111-119 